\title{
A Case Report on Capecitabine Induced Hand Foot Syndrome and Mucositis
}

\author{
Ponni Jothi Prakasam ${ }^{* 1}$, Divya Gopala Krishnan', Guru Prasad Mohanta ${ }^{2}$ \\ ${ }^{1}$ Department of Pharmacology, Government Medical College Calicut- 673008, Kerala, India \\ 2Department of Pharmacy Practice, Annamalai University, Chidambram-608002, Tamil Nadu, India.
}

\begin{abstract}
Hand foot syndrome (HFS) is a dose-limiting toxicity of Capecitabine. Though HFS is not life threatening, it can affect the daily activities and quality of life of a patient. Proper patient education aids in early detection of HFS and in minimizing or preventing the harm caused by it. We report a case of an elderly male patient who received adjuvant chemotherapy with Capecitabine for advanced Gastric carcinoma. While on treatment, he developed signs and symptoms consistent with Capecitabine induced grade 2 HFS and oral mucositis. The adverse drug reaction causality assessment done using the WHO scale showed a 'Probable' association of the reaction with Capecitabine.
\end{abstract}

Key words: Capecitabine, Gastric carcinoma, Hand foot syndrome, Mucositis, Patient education.

\section{INTRODUCTION}

Hand Foot Syndrome (HFS), also known as "Palmoplantar erythrodysesthesia, Acral erythema, or Burgdorf's reaction", is a cutaneous reaction caused by certain chemotherapeutic agents that manifests as varying degrees of dysesthesia, painful erythema, edema and desquamation of palms and soles, which though not life threatening increases the morbidity of the patient. 5-Fluorouracil (5FU) and its oral prodrug Capecitabine, Cytarabine, Doxorubicin, Epirubicin, Fluorode oxyuridine, Hydroxyurea, Mercaptopurine, Cyclophosphamide, Docetaxel and tyrosine kinase inhibitors including Sorafenib and Sunitinib are the frequently involved agents causing HFS. ${ }^{1,2}$

Capecitabine with the advantage of oral administration is currently indicated for treatment of Colorectal cancer, Breast cancer and advanced Upper Gastrointestinal cancer. ${ }^{3}$ HFS is a well defined adverse effect of Capecitabine; others being nausea, vomiting, oral mucositis, diarrhea and myelo- suppression. ${ }^{4}$ Treatment of Capecitabine induced HFS is immediate interruption of the drug and providing symptomatic care to the patient and therefore resolution of symptoms occur. ${ }^{5}$

We report a case of HFS and oral mucositis in a patient of advanced gastric cancer treated with Capecitabine. Our aim is to lay emphasis on the significance of patient education at the start and during therapy thus attempting to prevent common and predictable ADRs.

\section{Case Report}

A 65 year old male patient diagnosed with advanced gastric cancer with gastric outlet obstruction underwent palliative Gastrojejunostomy and was started on adjuvant chemotherapy with oral Capecitabine 500 mg twice daily for 14 days followed by a 7 day tablet free interval. The treatment was home based and he was asked to review on the $21^{\text {st }}$ day after start of treatment. On
DOI: 10.5530/ijopp.7.4.9

Address for correspondence: Dr. Ponni Jothi Prakasam Technical Associate, ADRMonitoring Centre, PvPI Department of Pharmacology,GMC-Calicut, 673008, Kerala. Email:ponnij87@yahoo. co.in

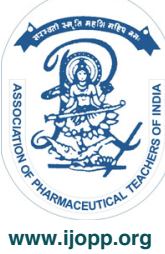




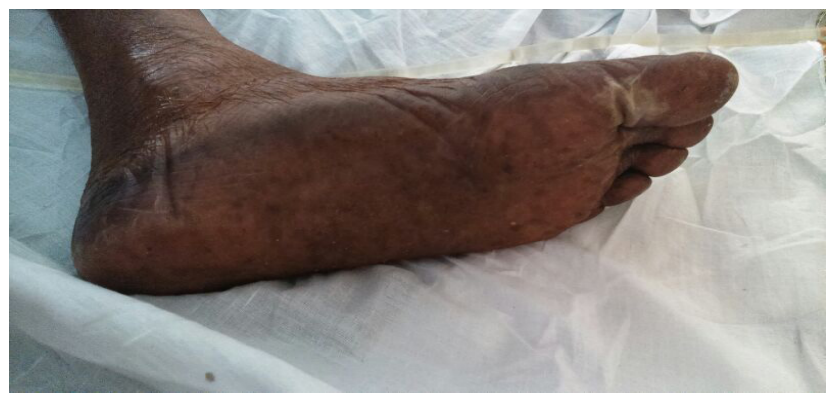

Figure 1: Discolored rashes and peeling of skin on sole

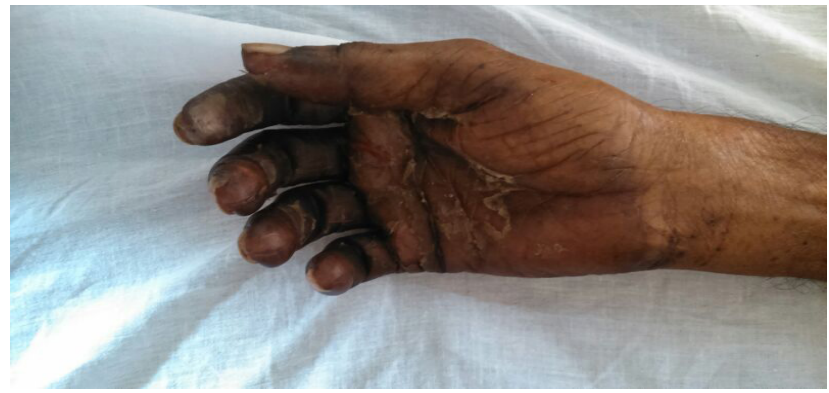

Figure 2: Discolored rashes and peeling of skin on palm

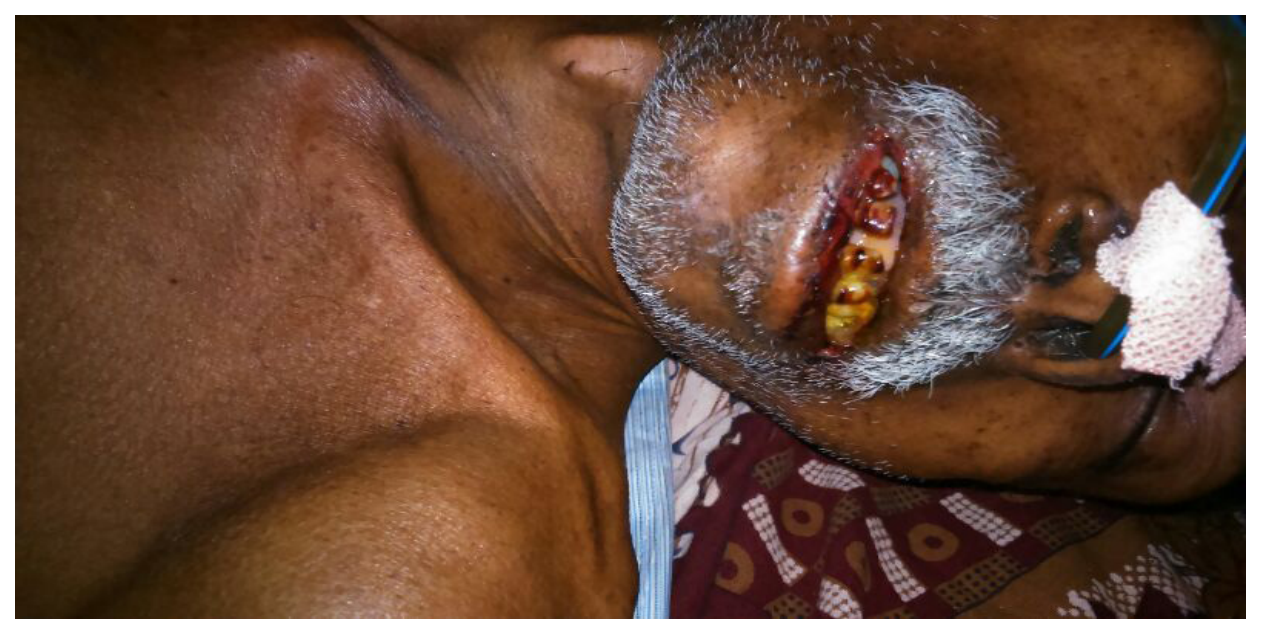

Figure 3: Oral mucositis

the eighth day after initiating therapy, the patient experienced tingling sensation in both his palms. Over the next two days, he developed erythema on his palms first and then on the soles. The patient ignored the symptoms which progressed to burning type of pain, blackish discoloration with peeling of skin over the affected areas in the next 4 days. Also during these 4 days, he developed painful oral erosions. It was then that patient became incapacitated from doing his daily activities and also his food intake became difficult. He then reported to our tertiary centre on the $15^{\text {th }}$ day after start of treatment by which time he had received the full course of Capecitabine for the cycle. Dermatological examination of the patient on admission showed discolored rashes with fissuring and desquamation of skin over both palms and soles which was more severe on palms and interfered with hand functions (Figure 1 and 2). Oral examination showed painful erosions on the lips and buccal mucosa (Figure 3). The skin on the other parts of his body was normal.

Vital signs and systemic examination of the patient were within normal limits. Laboratory tests for complete blood count, electrolytes, renal function tests, liver function tests were within normal limits. He was diagnosed as a case of Capecitabine induced Grade 2 HFS and mucositis based on clinical judgment. The adverse drug reaction causality assessment done using the WHO scale showed a 'Probable' association of the reaction with Capecitabine. He was managed with prophylactic antibiotics (Cefotaxime and Metronidazole) to prevent secondary infection of the skin lesions, topical emollients (liquid paraffin), oral steroids, Vitamin $\mathrm{E}$ and B complex and Non-steroidal anti-inflammatory drug (Diclofenac). Symptoms of the patient improved over 7 days of hospital stay. He was then discharged with advice to continue topical emollients and multivitamins.

\section{DISCUSSION}

Capecitabine is a systemic prodrug of $5 \mathrm{FU}$ which is currently FDA approved for use as adjuvant therapy in Colorectal cancer and Gastric cancer, first line therapy in metastatic Colorectal cancer and metastatic Breast cancer. Capecitabine as compared to 5FU has the added advantage of oral administration making it expedient in home based setting, thereby minimizing need for venous access as well as hospital stay. ${ }^{3}$

According to one of the recent study incidence of grade 3 HFS, among four capecitabine-based chemotherapy regimens [cisplatin / capecitabine (CX), epirubicin / cisplatin / capecitabine (ECX), epirubicin / oxaliplatin / capecitabine (EOX) and docetaxel / cisplatin / capecitabine (DCX)] as first-line treatment for advanced and/or metastatic gas- 
Table 1: Grading of HFS according to severity as per CTCAE Version 4.03

\begin{tabular}{lll}
\hline GRADE 1 & GRADE 2 & GRADE 3 \\
\hline Minimal skin changes & Skin changes & Severe skin \\
or dermatitis (e.g. & (e.g. peeling, & changes \\
erythema, edema, & blisters, edema & (e.g. peeling, \\
hyperkeratosis without & or hyperkeratosis & blisters, \\
pain) & with pain limiting & bleeding, \\
& instrumental ADL) & $\begin{array}{l}\text { hyperkeratosis } \\
\text { with pain } \\
\end{array}$ \\
& & limiting self \\
& & care ADL) \\
\hline
\end{tabular}

ADL=Activities of daily living
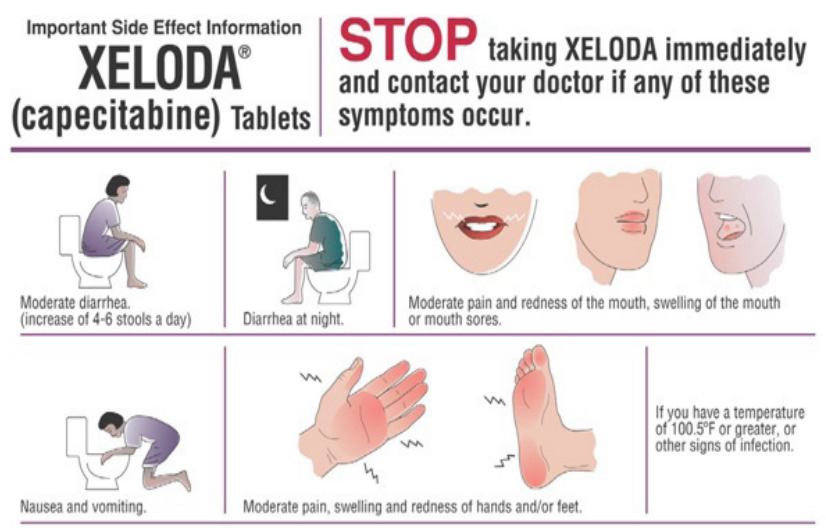

- If caught early, most of these side effects usually improve after you stop taking XELODA. - If they do not improve within 2 to 3 days, call your doctor again.

- After side effects have improved, your doctor will tell you whether to start taking XELODA again or what dose to use.

Figure 4: Pictogram depicting side effect of Capecitabine

tric cancer were $6.3,5.2,3.7$ and $2.4 \%$ respectively out 108 patients requiring therapy. ${ }^{6}$

In a second study involving pooling of two phase 3 clinical trials comprising 596 patients, where Capecitabine monotherapy was first line drug for metastatic colorectal cancer, incidence of all the grades HFS was found to be $54 \%{ }^{7}$

HFS is a dose limiting adverse effect of Capecitabine. ${ }^{4}$ Pathphysiology of Capecitabine induced HFS is less known. Certain theories suggest that Capecitabine may be eliminated by the eccrine glands, the resulting excretion causing HFS, since the hands and feet have an increased number of eccrine glands. ${ }^{8}$ Histopathological changes of HFS include vacuolar degeneration of basal keratinocytes, dermal per vascular lymphocytic infiltration and dermal edema. ${ }^{9}$ Some theories even suggest that it is the result of an inflammatory reaction due to over expression of COX-2. ${ }^{10,11}$

HFS is a threat to the quality of life of patients due to the extent it limits their physical activities. Accord- ing to Clinical Terminology Criteria for Adverse Events (CTCAE) version 4.0, HFS is classified into 3 grades based on the severity ${ }^{12}$ (Table 1). Treatment of Capecitabine induced HFS is immediate discontinuation of the drug and supportive measures to reduce pain and to prevent secondary infection. On resolution of symptoms, the drug is restarted at the same dose or reduced dose depending on the severity and frequency of the adverse effect. ${ }^{5}$

Proper patient education by the treating physician prior to the start of medication and early recognition of symptoms can help in preventing/minimizing this adverse effect. In our case, lack of awareness of the patient of the potential adverse effect and the ways to prevent it led to the progression of his symptoms. The health care professionals concerned should inform the patients of this adverse effect and should instruct them to avoid activities causing friction to hands and legs, to wear sunscreen to protect the skin, to avoid ill fitting shoes, to avoid extreme temperatures, to use mild soap and lukewarm water for bath and to use moisturizing creams or emollients frequently to keep skin moist. They should be instructed to report immediately to the physician in case they notice mouth ulcer, itching or redness of skin over hand or foot. ${ }^{13}$ Pictograms depicting the possible side effects of Capecitabine will visually aid patients to remember and to act promptly by informing their health care providers in case of early signs of HFS. Example of such a pictogram is shown in Figure $4{ }^{7}$

\section{CONCLUSION}

Motivation and awareness on the part of both health care professionals and patients can go a long way in minimizing such predictable adverse effects.

\section{CONFLICT OF INTEREST}

The authors declare no conflicts of interest

\section{ACKNOWLEDGMENTS}

We would like to sincerely thank Dr. K. Kalaiselvan, Principal Scientific officer, IPC, Pharmacovigilance Programme of India for the support and Department of Pharmacology-GMC Kozhikode for continuous encouragement. We would like to extent our gratitude to the staff of Department of Medicine, GMC, Calicut for their support. We are also thankful to the patient for his co-operation. 


\section{REFERENCES}

1. Degen A, Alter M, Schenck F, et al. The hand-foot syndrome associated with medical tumor therapy - classification and management. J Dtsch Dermatol Ges. 2010; 8(9): 652-61

2. Lipworth AD, Robert C, Zhu AX. Hand-foot syndrome (hand-foot skin reaction, palmar-plantar erythrodysesthesia: Focus on sorafenib and sunitinib. Oncology 2009; 77(5): 257-71.

3. Schellens JHM. Capecitabine. The Oncologist February 2007; 12(2): 152-5.

4. Saif MW, Kartitzoglou NA, Syrigos KN. Capecitabine: an overview of the side effects and their management. Anticancer drugs 2008 Jun; 19(5): 447-64.

5. Lassere $Y$, Hoff P. Management of hand-foot syndrome in patients treated with capecitabine (Xelodas). European Journal of Oncology Nursing 2004; 8 (suppl 1), $\$ 31-40$

6. Gomez-Martin C, et al. Incidence of hand-foot syndrome with capecitabine in combination with chemotherapy as first-line treatment in patients with advanced and/or metastatic gastric cancer suitable for treatment with a fluoropyrimidine-based regimen. Clin Transl Oncol. 2012, Sep; 14(9): 689-97.

7. http://www.gene.com/patients/medicines/xeloda accessed on 11/12/14
8. Baack BR, Burgdorf WH. Chemotherapy induced acral erythema. J Am Acad Dermatol. 1991; 24(3): 457-61

9. Mrozek-Orlowski ME, Frye DK, Sanborn HM. Capecitabine: nursing implications of a new oral chemotherapeutic agent. Oncol Nurs Forum 1999; 26(4): 753-62

10. Narasimhan P, Narasimhan S, Hitti IF, Rachita M. Serious hand-and-foot syndrome in black patients treated with Capecitabine: report of 3 cases and review of the literature. Cutis 2004; 73(2): 101-6.

11. Lin E, Morris JS, Ayers GD. Effect of Celecoxib on Capectabine-induced hand-foot syndrome and antitumor activity. Oncology (Williston Park 2002; 16 (12 Suppl No 14):31-7

12. National Cancer Institute, Cancer Therapy Evaluation Program.Common Terminology Criteria for Adverse Events v4.03 (CTCAE). http://evs.nci.nih.gov/ftp1/CTCAE/ CTCAE_4.03_2010-06 14_QuickReference_8.5x11.pdfaccessed on 17/08/14

13. Muhammad Wasif Saif, Aymen A. Elfiky, Identifying \& Treating fluoropyrimidine associated hand foot syndrome in White \&Non white patients. Supportive oncology 2007; 5(7): 337-43.and/or metastatic gastric cancer suitable for treatment with a fluoropyrimidine-based regimen. Clin Transl Oncol. 2012, Sep; 14(9): 689-97. 